\title{
Self Heating during Stretch Blow Molding: an Experimental Numerical Comparison
}

\author{
Yun-Mei Luo ${ }^{1, a)}$, Luc Chevalier ${ }^{1, b)}$, Eric Monteiro ${ }^{2, c)}$ and Françoise Utheza ${ }^{1, d)}$ \\ ${ }^{1}$ Université Paris-Est Marne-La-Vallée, Laboratoire Modélisation et Simulation Multi Echelle, MSME UMR 8208 \\ CNRS, 5 bd Descartes, 77545 Marne-la-Vallée, France \\ ${ }^{2}$ Arts et Métiers ParisTech-PIMM, UMR CNRS 8006, 151 Bd de l'Hôpital, 75013 Paris \\ a)yunmei.luo@univ-paris-est.fr \\ b) luc.chevalier@univ-paris-est.fr \\ ${ }^{c}$ eric.monteiro@ensam.eu \\ d) francoise.utheza@univ-paris-est.fr
}

\begin{abstract}
The great influence of temperature on polymer's behavior is well known and, for example, a $10^{\circ} \mathrm{C}$ increase can lead to a 10 time reduction of the viscosity. The necessity to take into account the self heating phenomena appears then to be crucial for thermoforming process simulation and in particular for ISBM of PET. An original anisotropic viscohyperelastic model coupled with temperature has been developed [1,2], identified from equibiaxial and constant width tension tests [3] and can be used to evaluate this self-heating phenomenon. In order to provide validation of the model and its capacity to predict accurately final bubble shape, self-heating value and induced mechanical properties, free blowing of a preform has been carried out with temperature measured using a thermal camera. Results are discussed and compared with numerical simulation prediction. At room temperature, tension specimens have been cut from the different blown bubbles and induced modulus where measured in three directions: longitudinal and hoop directions and also $45^{\circ}$ from both previous ones and an elastic orthotropic behavior is identified. The effect of the self heating on the free blown shapes and induced modulus is discussed.
\end{abstract}

\section{INTRODUCTION}

The injection stretch blow molding (ISBM) process which is managed at a temperature slightly above the glass transition temperature Tg involves multiaxial large strains at high strain rate of polyethylene terephthalate material (PET). The performance of a PET bottles produced by the ISBM process depends on many parameters. During the ISBM process, the PET behavior exhibits high elasticity, a strain hardening effect and a strong viscous and temperature dependency. Therefore, much research has been conducted on the rheological behavior of PET. Essentially, the viscoelastic model which take into account the strain hardening and strain rate effects have been widely used for ISBM process in literature [1,2]. Effects of temperature (initial heating conditions and self-heating), are of fundamental importance during the injection stretch blow moulding process of PET bottles. During the ISBM process, an initial preform is firstly heated in an oven to the process temperature, which is near or slightly above Tg. After that, during the process, the low mobility of micromolecules and the high viscosity of the material generate dissipation of energy which leads to a self-heating phenomenon. This self-heating effect must be taken into account in the simulation. In contrast, many existing numerical studies of ISBM [2,3] neglect heat transfer: the temperature has been assumed to be constant during the process and the heat induced by deformation was neglected. Schmidt et al. [4], one of the first to investigate self-heating simulation, developed a non-isothermal finite element ISBM simulation [5] to embed heat transfer during the deformation process. We proposed a non linear incompressible visco-hyperelastic model to reproduce the complex constitutive behavior of PET [6,7]. Considering the thermal aspects, an experimental study and the numerical thermo-viscoelastic simulation for the 2D PET sheets have been performed[8] 
First, in the numerical part, based on the experimental data of biaxial tension tests, we identified the properties of the proposed visco-hyperelastic orthotropic behavior taking into account the self-heating effect in the identification process. In this work, in order to process closer the case of industrial reality and to benefit from the opportunities of $\mathrm{CAD}$ and mesh construction software, the model is implemented into the software ABAQUS / Explicit via a userinterface VUMAT. The free blowing simulation, taking into account the anisotropy and the effect of the temperature, is performed.

In the second section of this work, an experimental study for the free blow case is performed. An infrared camera is used to capture the temperature field evolution during free blowing of a PET preform. Different initial temperature with the same air rate and max pressure conditions are performed to obtain the bottles. The self-heating phenomenon is observed. Both numerical and experimental results for the increment of the temperature from dissipation of energy are compared. Moreover, for each bottle more than 10 specimen were cut for longitudinal, hoop and $45^{\circ}$ directions, related modulus and Poisson's ratio were measured and mean values are shown. An elastic orthotropic behavior is identified from these uni-axial tension specimen at room temperature.

\section{NUMERICAL IDENTIFICATION AND SIMULATION OF FREE BLOWING}

The proposed non linear incompressible visco-hyperelastic model on isotropic version has been presented and identified in the author's previous papers [6,7]. In order to obtain the correct aspect ratio between length and radius during the free blowing simulation or to reproduce accurately the constant width test, one needs to introduce anisotropy in both the viscous and elastic parts of the model. The strongly hyperelastic strain rate dependant and coupled with the temperature is modeled using a Maxwell like model in finite strain. The Cauchy stress tensor $\underline{\underline{\sigma}}$ can be written:

$$
\left\{\begin{array}{l}
\underline{\underline{\sigma}}=-P_{e} \underline{\underline{I}}+2 G \underline{\underline{\varepsilon_{e}}} \\
\underline{\underline{\sigma}}=-P_{v} \underline{\underline{I}}+2 \eta \underline{\underline{D_{v}}}
\end{array}\right.
$$

where $\underline{\underline{\varepsilon_{e}}}$ is an Eulerian strain tensor: $\underline{\underline{\varepsilon_{e}}}=\frac{1}{2}\left(\underline{\underline{B_{e}}}-\underline{I}\right), P_{e}$ and $P_{v}$ are hydrostatic pressures associated to incompressibility conditions. $\underline{\underline{B_{e}}}$ is the elastic part of the left Cauchy deformation tensor.

Both the elastic and the viscous parts of the model must contribute to the strain rate effect.

\section{Elastic part Visco-Hyperelastic Model}

We can constitute a free energy function $W$ as a function of the invariants. The principal invariants of the elastic left Cauchy Green tensor which associated to the isotropic material behavior can be written as:

$$
I_{1}=\operatorname{tr}\left(\underline{\underline{B_{e}}}\right), \quad I_{2}=\frac{1}{2}\left[\underline{\operatorname{tr}} \underline{\left.\underline{B_{e}}\right)^{2}}-\operatorname{tr}\left(\underline{\underline{B_{e}^{2}}}\right)\right], \quad I_{3}=\operatorname{det}\left(\underline{\underline{B_{e}}}\right)
$$

In order to build the anisotropy in elastic part of the model, we need new invariants. The invariants associated with the anisotropic behavior, namely:

$$
\begin{aligned}
& I_{4}=\underline{n_{1}} \cdot \underline{\underline{B_{e}}} \cdot \underline{n_{1}}, \quad I_{6}=\underline{n_{2}} \cdot \underline{\underline{B_{e}}} \cdot \underline{n_{2}}, \quad I_{8}=\underline{n_{3}} \cdot \underline{\underline{B_{e}}} \cdot \underline{n_{3}}, \\
& I_{5}=\underline{n_{1}} \cdot \underline{\underline{B_{e}^{2}}} \cdot \underline{n_{1}}, \quad I_{7}=\underline{n_{2}} \cdot \underline{\underline{B_{e}^{2}}} \cdot \underline{n_{2}}, \quad I_{9}=\underline{n_{3}} \cdot \underline{\underline{B_{e}^{2}}} \cdot \underline{n_{3}}
\end{aligned}
$$

where $\underline{n_{1}}, \underline{n_{2}}$ et $\underline{n_{3}}$ are the privileged directions. Three second order structural tensors $\underline{A_{i}}$ can be calculated with the dyadic product of the preferred directions with themselves:

$$
\underline{\underline{A_{1}}}=\underline{n_{1}} \otimes \underline{n_{1}}, \quad \underline{\underline{A_{2}}}=\underline{n_{2}} \otimes \underline{n_{2}}, \quad \underline{\underline{A_{3}}}=\underline{n_{3}} \otimes \underline{n_{3}}
$$

The Hart Smith model is chosen to characterize the free energy function. Consequently, the energy function can be written as the following form:

$$
W\left(\underline{\underline{B}}, \underline{\underline{A_{1}}}, \underline{\underline{A_{2}}}, \underline{\underline{A_{3}}}\right)=G_{1} \int_{0}^{I_{1}-3} e^{\Lambda_{1} X^{2}} d X+G_{2}\left(\int_{0}^{I_{4}-1} e^{\Lambda_{2} X^{2}} d X+\int_{0}^{I_{6}-1} e^{\Lambda_{2} X^{2}} d X\right)
$$

Hence, the Cauchy stress can be calculated from the strain energy: 
where $W_{i}=\partial W / \partial I_{i}$

$$
\begin{aligned}
\underline{\underline{\sigma}}=2 \frac{\partial W}{\partial B_{e}} & =-p_{e} \underline{\underline{I}}+2\left(W_{1}+I_{1} W_{2}\right) \underline{\underline{B_{e}}}-2 W_{2} \underline{\underline{B_{e}^{2}}} \\
& +2 I_{4} W_{4} \underline{\underline{A_{1}}}+2 I_{4} W_{5}\left(\underline{\underline{n_{1}} \otimes} \underline{\underline{B_{e}}} \underline{\underline{n_{1}}}+\underline{n_{1}} \underline{\underline{B_{e}}} \otimes \underline{n_{1}}\right) \\
& +2 I_{6} W_{6} \underline{\underline{A_{2}}}+2 I_{6} W_{7}\left(\underline{\underline{n_{2}} \otimes B_{e}} \underline{\underline{n_{2}}}+\underline{n_{2}} \underline{\underline{B_{e}}} \otimes \underline{n_{2}}\right) \\
& \left.+2 I_{8} W_{8} \underline{\underline{\underline{A_{3}}}}+2 I_{8} W_{9} \underline{\underline{n_{3}} \otimes} \underline{\underline{B_{e}}} \underline{\underline{n_{3}}}+\underline{\underline{n_{3}}} \underline{\underline{B_{e}}} \otimes \underline{n_{3}}\right)
\end{aligned}
$$

After the application of the model choice for the energy function $W$ (Eq.5), the elastic stress yields to:

$$
\underline{\underline{\sigma}}=-p \underline{\underline{I}}+2 G_{1} e^{\Lambda_{1}\left(I_{1}-3\right)^{2}} \underline{\underline{B}}+2 I_{4} G_{2} e^{\Lambda_{2}\left(I_{4}-1\right)^{2}} \underline{\underline{A_{1}}}+2 I_{6} G_{2} e^{\Lambda_{2}\left(I_{6}-1\right)^{2}} \underline{\underline{A_{2}}}
$$

where $G_{1}, G_{2}, \Lambda_{1}$ and $\Lambda_{2}$ are parameters in elastic part of the Visco-hyperelastic model.

\section{Viscous part Visco-Hyperelastic Model}

The deviatoric part of the stress tensor $\hat{\underline{\hat{\sigma}}}$ in our case can be written:

$$
\hat{\underline{\sigma}}=2 \underline{\underline{\underline{\eta}}} \underline{\underline{\underline{D_{v}}}} \text { that also writes: }\left(\begin{array}{c}
\hat{\sigma}_{11} \\
\hat{\sigma}_{22} \\
\sqrt{2} \sigma_{12}
\end{array}\right)=2\left[\begin{array}{ccc}
\eta_{11} & \eta_{12} & 0 \\
\eta_{12} & \eta_{22} & 0 \\
0 & 0 & \eta_{44}
\end{array}\right]\left(\begin{array}{c}
D_{v 11} \\
D_{v 22} \\
\sqrt{2} D_{v 12}
\end{array}\right)
$$

where $D_{v}$ is the viscous strain rate. We choose specific $h_{i}$ functions for each orthotropic direction (i=1 the hoop direction and $\mathrm{i}=2$ longitudinal direction):

$$
\left\{\begin{array}{c}
\eta_{11}=\eta_{0}(T) h_{1} f\left(\left(\dot{\bar{\varepsilon}}_{v}\right)\right. \\
\eta_{12}=\beta \eta_{0}(T) \max \left(h_{1}, h_{2}\right) h f\left(\dot{\bar{\varepsilon}}_{v}\right) \\
\eta_{22}=\eta_{0}(T) h_{2} f\left(\frac{\dot{\varepsilon}_{v}}{\dot{\varepsilon}_{v}}\right) \\
\eta_{44}=\eta_{0}(T) h\left(\bar{\varepsilon}_{v}\right) f\left(\frac{\dot{\varepsilon}_{v}}{\dot{\varepsilon}_{v}}\right)
\end{array},\left\{\begin{array}{l}
h_{1}=\left(1-\exp \left(-K \varepsilon_{v 1}\right)\right) \cdot \exp \left(\alpha_{1}\left(\frac{\varepsilon_{v 1}}{\varepsilon_{v r e f}(T)}\right)^{2}+\alpha_{2} \varepsilon_{v 1}+\alpha_{3}\right) \\
h_{2}=\left(1-\exp \left(-K \varepsilon_{v 2}\right)\right) \cdot \exp \left(\alpha_{1}\left(\frac{\varepsilon_{v 2}}{\varepsilon_{v r e f}(T)}\right)^{2}+\alpha_{2} \varepsilon_{v 2}+\alpha_{3}\right)
\end{array}\right.\right.
$$

with

$$
\begin{gathered}
f\left(\overline{\dot{\varepsilon}}_{v}\right)=\frac{1}{\left(1+\left(\lambda \overline{\dot{\varepsilon}}_{v} / \dot{\varepsilon}_{r e f}\right)^{a}\right)^{\frac{1-m}{a}}} \\
\ln \left(\alpha_{T}\right)=\frac{-C_{1}\left(T-T_{r e f}\right)}{C_{2}+T-T_{r e f}}, \eta_{0}(T)=\alpha_{T} \eta_{0}\left(T_{r e f}\right) \\
\varepsilon_{v r e f}(T)=\frac{\varepsilon_{v r e f}\left(T_{r e f}\right)}{\exp \left(-C_{3}\left(T-T_{r e f}\right)+C 4\right)}
\end{gathered}
$$

where $\lambda, m, a$ are parameters in the Carreau type law $f\left(\overline{\dot{\varepsilon}}_{v}\right)$ and $\dot{\varepsilon}_{r e f}$ is a reference strain rate that can be taken equal to $1 \mathrm{~s}^{-1}$ for sake of simplicity. $\beta$ is an parameter indicate the difference of the beginning of the hardening effect between equal biaxial and constant width tension test. $\mathrm{K}, \alpha_{1}, \alpha_{2}$ and $\alpha_{3}$ are parameters in $h$ function which can be identified from biaxial elongation tests [9]. Variables $\eta_{0}(T)$ and $\varepsilon_{\text {vref }}(T)$ show a significant dependence on temperature. We choose the Williams-Landel- Ferry (WLF) model for the evolution of $\eta_{0}(T) . C_{1}$ and $C_{2}$ are the WLF parameters, $T_{r e f}=90^{\circ} C . C_{3}$ and $C_{4}$ are parameters in the expression of $\mathcal{E}_{\text {vref }}(T)$.

Therefore, the equation8 for the deviatoric part of the stress can be written as:

$$
2 \underline{\underline{\eta}} \underline{\underline{\underline{D_{v}}}}=2 G_{1} e^{\Lambda_{1}\left(I_{1}-3\right)^{2}} \underline{\underline{B}}+2 I_{4} G_{2} e^{\Lambda_{2}\left(I_{4}-1\right)^{2}} \underline{\underline{A_{1}}}+2 I_{6} G_{2} e^{\Lambda_{2}\left(I_{6}-1\right)^{2}} \underline{\underline{A_{2}}}
$$




$$
\Rightarrow\left(\begin{array}{c}
D_{v 11} \\
D_{v 22} \\
D_{v 12}
\end{array}\right)=\left[\begin{array}{ccc}
\frac{\eta_{22}}{\eta_{11} \eta_{22}-\eta_{12}^{2}} & \frac{-\eta_{12}}{\eta_{11} \eta_{22}-\eta_{12}^{2}} & 0 \\
\frac{-\eta_{12}}{\eta_{11} \eta_{22}-\eta_{12}^{2}} & \frac{\eta_{22}}{\eta_{11} \eta_{22}-\eta_{12}^{2}} & 0 \\
0 & 0 & \frac{1}{\eta_{44}}
\end{array}\right]\left(\begin{array}{c}
\tilde{d}_{11} \\
\tilde{d}_{22} \\
\tilde{d}_{12}
\end{array}\right)
$$

where $\left(\begin{array}{c}\tilde{d}_{11} \\ \tilde{d}_{22} \\ \tilde{d}_{12}\end{array}\right)$ is the vector form for the tensors from the right side of the equation 14.

In the following, the equation 14 can be used to identify the parameters from the tension test and simulate the ISBM process. From the identified parameters one can plot figure 1(b)and compare the numerical and experimental results. The mean difference does not exceed $15 \%$.
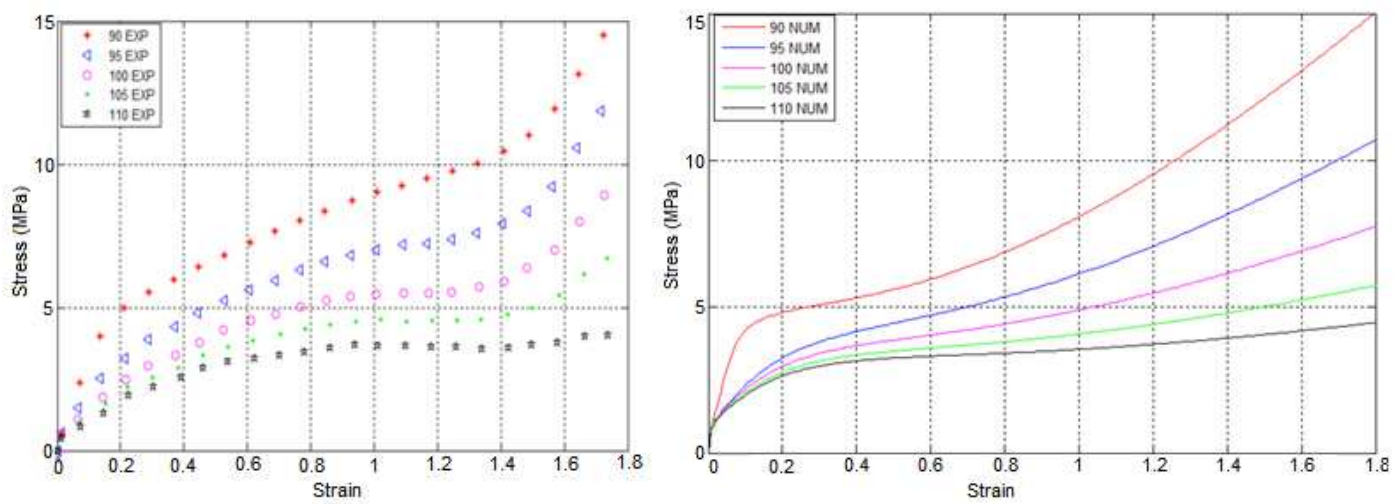

FIGURE 1. Comparison between experimental results and model simulations

The model is implemented into the software ABAQUS / Explicit via a user interface VUMAT. This strongly nonlinear problem is solved using a classical Newton Raphson iterative procedure. We performed a thermomechanical simulation. The dissipation of energy is obtained by $\sigma: D_{v}$. Considering the time needed for blowing, we assume the conduction effect is neglected in regard of the dissipation effect. Therefore, the thermal part can be written as follow:

$$
\rho C_{p} \dot{T}=\sigma: D_{v}
$$

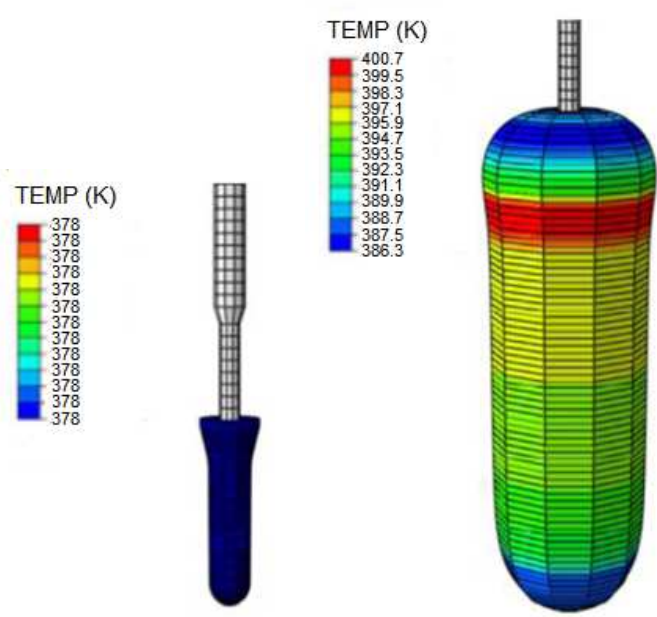

FIGURE 2.Finite element simulation of free blowing (left) initial preform and rod, (b) blown preform and temperature field where one can see the increase of temperature due to deformation. 
We assume that the major contribution to the self heating phenomenon is due to viscous dissipation. The entropy of rubber networks convection or crystallization is neglected.

We choose $\rho=1330 \mathrm{~kg} \cdot \mathrm{m}^{-3}$ and $C_{p}=1200 \mathrm{~J} \cdot \mathrm{kg}^{-1} \cdot \mathrm{K}^{-1}$. Figure 2 shows the temperature distribution of the initial preform and of the bottle. One can see that the increase of temperature due to the self heating phenomenon can reach $20^{\circ} \mathrm{C}$.

\section{EXPERIMENTAL MEASUREMENTS: SELF HEATING AND INDUCED MODULUS}

In order to provide validation of the model and its capacity to predict accurately final bubble shape, self-heating value and induced mechanical properties, free blowing of preform has been carried out with temperature measured using a thermal camera. A FLIR B250 infrared camera with the wavelength range $7.5-13 \mu \mathrm{m}$ is used to evaluate the temperature distribution. The polymer material is opaque under the wavelength in the range of $8-12 \mu \mathrm{m}$. We assume that the emissivity value is constant.

The free blowing at different initial temperature with the same max pressure conditions ( 8 bar) are carried out. Figure $3 \mathrm{a}$ and $3 \mathrm{~b}$ shows the temperature measurements during free blowing of PET preform. For a given initial temperature, one can see the increase of temperature can reach about $20^{\circ} \mathrm{C}$. There is an agreement with experimental and numerical results. The increases of the temperature do not vary significantly from different zone and different initial temperature: the mean difference does not exceed $22 \%$.

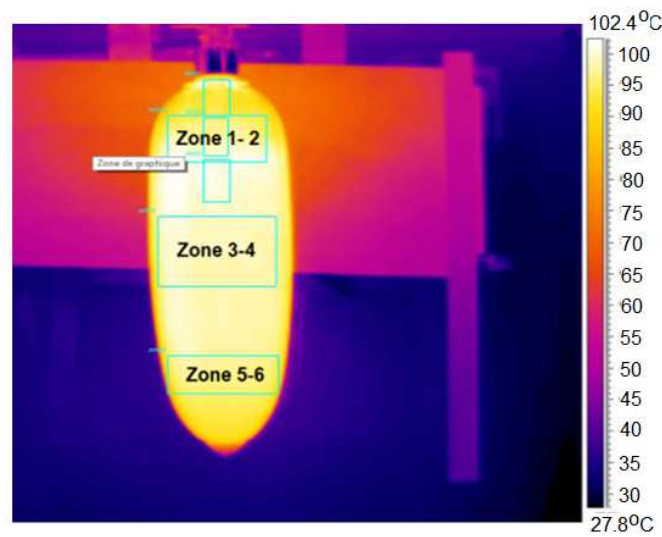

\begin{tabular}{|l|r|r|r|r|}
\hline${ }^{\circ}{ }^{\circ} \mathrm{C}$ & 90 & 95 & 100 & 105 \\
\hline zone 1-2 & 16,04 & 17,97 & 17,92 & 18,117 \\
\hline zone3-4 & 16 & 18,27 & 17,529 & 18,285 \\
\hline zone 5-6 & 17,53 & 19,94 & 21,84 & 18,221 \\
\hline
\end{tabular}

(a)

(b)

FIGURE 3. Temperature measurements during free blowing of PET preform. (a) Snap shot of the thermal field and location of measurement area. Tight rectangles give initial temperature of the preform and large ones give the corresponding final temperature. (b) 3 different areas are chosen to follow the increase of the mean temperature in one specific bottle

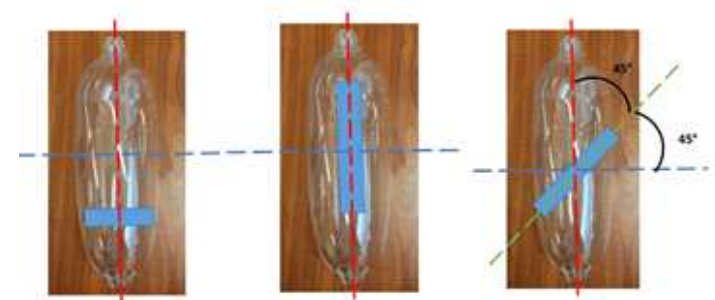

(a)

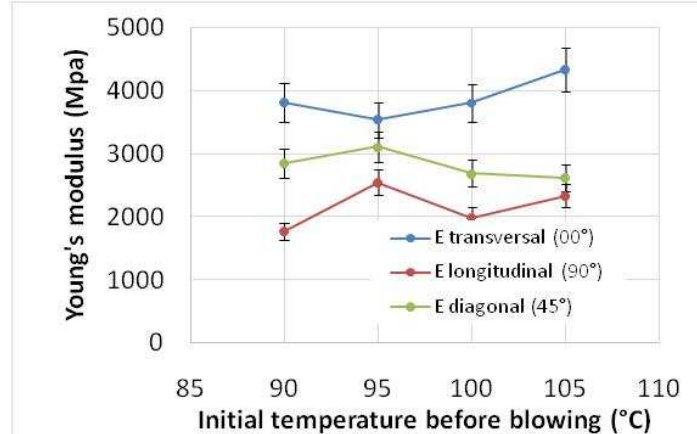

(b)

FIGURE 4. Anisotropic induced modulus measurement from uni-axial tension specimen cut out from blown bottle. (a) The bottle is cut for 3 directions to measure modulus. (b) Summary of measured modulus. For each bottle more than 10 specimen were cut for each direction, related modulus and error bars are shown. The dispersion defined by the ratio standard deviation over mean value is equal to $8 \%$ (mean dispersion for all measures). 
From figure $4 \mathrm{~b}$, we see that this similarity of self heating as consequence on elastic orthotropic behavior of the bottle back to room temperature. Modulus $\mathrm{E}$ and Poisson ratio are measured and mean values of each bottle is used to identify the 5 parameters $E_{1}, E_{2}, v_{12}, v_{21}$ and $G_{12}$ from 6 experimental data and one more symmetry condition. One can see that is no particular tendency for the evolution of Young's modulus for different initial temperature of the preform. This observation could be rather troubling because the higher is the preform temperature, the more the internal mobility of the macromolecular chains will be possible. Furthermore, a stretching step carried out at "high" temperature will produce a lower molecular elongation than at low temperature due to the relaxation of the material. For this reason, we expected to see all the modulus decrease with the temperature but this is not the case.

Figure $3 \mathrm{~b}$ shows that the self-heating does not seem to evolve with initial temperature for a given area. Indeed, self-heating arises from the dissipation of permanent deformation energy (here the viscous deformation), but this energy is in proportion of the product $\eta D^{2}$. The viscosity $\eta$ decreases when temperature increases and consequently, for a given pressure, the strain rate $D$ increases. The self heating tendency is then not so easy to predict. To improve the study and lead to more convincing results, we should make sure that all the bottles are stretched in the same way (same final volume). This necessitates reducing the flow rate and / or the pressure to make sure that all blowing are made with the same mean strain rate in order to allow an accurate comparison on the different specimen and highlight the influence of the initial temperature on the self-heating phenomenon and on induced properties.

\section{CONCLUSION}

The proposed non linear thermo-visco-hyperelastic model was identified from the equal biaxial tension results. This model was implemented in Abaqus to perform the thermo-mechanical simulation of the free blowing test. Due to this self heating simulation, a $20^{\circ} \mathrm{C}$ increased of temperature was obtained.

An experimental measurement was carried out with temperature measured using a thermal camera. Results are discussed and compared with numerical simulation prediction. Furthermore, the blown bottles have been cut and induced modulus where measured in three directions: longitudinal and hoop directions and also $45^{\circ}$ from both previous ones and an elastic orthotropic behavior is identified. The effect of the self heating on the free blown shapes and induced modulus is discussed.

\section{REFERENCES}

1. C.P. Buckley, D.C. Jones and D.P. Jones: "Hot Drawing of Poly(ethylene terephthalate) under Biaxial Stress: Application of a Three-Dimensional Glass-Rubber Constitutive Model". Polymer 37, 2403-2414, 1996.

2. C. W. Tan, G.H. Menary, Y. Salomeia, C. G. Armstrong, M. Picard, N. Billon, E.M.A. Harkin-Jones, P.J. Martin, K. Maheshwari. "Modelling of the Injection Stretch Blow Moulding of PET Containers via a PressureVolume-time (PV-t) Thermodynamic Relationship".International Journal of Material Forming, 1:799-802, 2008

3. G.H. Menary, C.G. Armstrong, R.J. Crawford, and J.P. McEvoy. "Modelling of poly(ethylene terephthalate) in injection stretch-blow moulding." Rubber and CompositesProcessing and Applications, 29, 360-370 (2000).

4. F.M. Schmidt, J.F. Agassant, and M. Bellet, "Experimental study and numerical simulation of the injection stretch/blow molding process."Polym. Eng. Sci., 38(9), 1399, 1998.

5. F.M.Schmidt, J.F. Agassant, M. Bellet, L. Desoutter, "Viscoelastic simulation of PET stretch/blow molding process." Journal of Non-Newtonian Fluid Mechanics, 64 (1), 19-42, 1996.

6. L. Chevalier; Y.M. Luo; E. Monteiro; G.H. Menary. "On visco-elastic modelling of polyethylene terephthalate behavior during multiaxial elongations slightly over the glass transition temperature."Mechanics of Materials, Vol. 52, p. 103-116, 2012.

7. Luo Y.M., Chevalier L., Monteiro E., "An Anisotropic Visco-hyperelastic model for PET Behavior under ISBM Process Conditions.", The 19th International ESAFORM Conference on Material Forming, Nantes, France, April 27-29, 2016.

8. Luo Y.M., Chevalier L., Utheza F., Monteiro E.: Numerical simulation of the thermodependant visco hyperelastic behavior of polyethylene terephthalate near the glass transition temperature: prediction of the selfheating during biaxial tension test. Polym. Eng. Sci.53, 2683-2695 (2013) 
9. Menary G.H., Tan C.W., Harkin-Jones E.M.A., Armstrong C.G., Martin P.J. "Biaxial Deformation of PET at Conditions Applicable to the Stretch Blow Molding Process". Polymer Engineering \& Science, vol. 52, no3, pp. 671-688, 2012. 